\title{
Noise-sustained structures in coupled complex Ginzburg-Landau equations for a convectively unstable system
}

\author{
M. Neufeld, ${ }^{1}$ D. Walgraef, ${ }^{1,2}$ and M. San Miguel ${ }^{1,3}$ \\ ${ }^{1}$ Departament de Física, Universitat de les Illes Balears, E-07071 Palma de Mallorca, Spain \\ ${ }^{2}$ Center for Nonlinear Phenomena and Complex Systems, Université Libre de Bruxelles, CP 231, B-1050 Brussels, Belgium \\ ${ }^{3}$ Instituto Mediterraneo de Estudios Avanzados, IMEDEA (CSIC-UIB), E-07071 Palma de Mallorca, Spain ${ }^{1 *}$
}

(Received 15 April 1996)

\begin{abstract}
We investigate a pattern-forming system close to a Hopf bifurcation with broken translational symmetry. In one-dimensional geometries, its evolution is governed by two coupled complex Ginzburg-Landau equations which describe the amplitude of the counterpropagating traveling waves that develop beyond the instability. The convective and absolute instabilities of the possible steady states are analyzed. In the regime of strong cross coupling, where traveling waves are favored by the dynamics, the results of previous analysis are recovered. In the weak cross-coupling regime, where standing waves are favored by the dynamics, traveling waves nevertheless appear, in the absence of noise, between the uniform steady state and the standing-wave patterns. In this regime, standing waves are sustained by spatially distributed external noise for all values of the bifurcation parameter beyond the Hopf bifurcation. Hence, the noise is not only able to sustain spatiotemporal patterns, but also to modify pattern selection processes in regimes of convective instability. In this weak coupling regime we also give a quantitative statistical characterization of the transition between deterministic and noise-sustained standing waves when varying the bifurcation parameter. We show that this transition occurs at a noise-shifted point and it is identified by an apparent divergence of a correlation time and the saturation of a correlation length to a value given by the system size. [S1063-651X(96)10412-8]

PACS number(s): 47.20.Ky, 02.50.Ey, 05.40,+j, 43.50.+y
\end{abstract}

\section{INTRODUCTION}

A series of physicochemical systems driven out of equilibrium undergo Hopf bifurcations with broken translational symmetries, which lead to the development of traveling or standing wave patterns. This is, for example, the case in Rayleigh-Bénard convection in binary or viscoelastic fluids [1], for spiral vortex flow in the Taylor-Couette system with counterrotating cylinders [2], or in electrohydrodynamic convection in liquid crystals [3]. Traveling rolls may also be obtained by the application of a through flow on hydrodynamic instabilities of the Rayleigh-Bénard or Taylor-Couette type [4-6]. As a result of the generic behavior of these systems in the vicinity of Hopf bifurcations, they may be described by coupled complex Ginzburg-Landau equations (CCGLE).

Effectively, it is now well known that, close to an instability, the spatiotemporal behavior of a system far from thermal equilibrium can be described by order-parameter-like equations [7-9]. The mathematical structure of these equations is rather universal and independent of the underlying physical system. The derivation of the order parameter equation from the basic evolution equations is made possible by the space-time separation between unstable and stable modes, and can be performed by different methods, such as adiabatic elimination of the stable, or "slaved" modes [8], or multiple scale analysis [9], for example.

In one-dimensional systems which undergo a Hopf bifurcation with broken spatial inversion symmetry, the order pa-

\footnotetext{
${ }^{1 * U R L: ~ h t t p: / / f o r m e n t o r . u i b . e s / N o n l i n e a r / ~}$
}

rameter field $\sigma(x, t)$ can be represented by two slowly varying envelope functions $A(x, t)$ and $B(x, t)$ for left and right traveling waves:

$$
\sigma(x, t)=A(x, t) e^{i\left(k_{c} x+\omega_{c} t\right)}+B(x, t) e^{-i\left(k_{c} x-\omega_{c} t\right)} .
$$

The evolution of the amplitude $A$ and $B$ is governed by CCGLE [7,10-14]. The nonlinear cross coupling between both amplitudes that determines if the stable patterns correspond to traveling (strong cross coupling) or standing (weak cross coupling) waves. The effect of the group velocity may usually not be discarded in the determination of the stability domain of the wave patterns. One has to distinguish between convective and absloute instability, and it is now well known that, sufficiently close to the Hopf bifurcation, the unpatterned state is convectively unstable but absolutely stable [5,13-17]. In this regime, localized perturbations are convected with the mean flow in such a way that they grow only in a moving reference frame but decay at any fixed location. On increasing the bifurcation parameter, one reaches a well defined threshold determined by the group velocity, and above which the reference state becomes absolutely unstable. In this regime, perturbations grow locally at fixed locations. As a result, the behavior of the system is qualitatively very different in both regimes. In the convectively unstable regime, a deterministic system cannot develop the expected wave patterns, except in special geometries, while in a stochastic system, noise is spatially amplified and gives rise to noise-sustained structures [15]. On the contrary, in the absolutely unstable regime, waves are intrinsically sustained by the deterministic dynamics.

Convectively unstable systems have been widely studied, both numerically and experimentally, but mostly in the case 
of single traveling waves. This situation is modeled by a single CGLE which emerges in the strong cross-coupling regime. In this case Deissler [15] obtained numerically noise sustained structures in the convectively unstable regime. Babcock et al. [5] and Tsamaret et al. [6] analyzed in detail the corresponding experimental situation in the case of a Taylor-Couette system with through flow. Both groups observed the transition from convective to absolute instability and were able to generate noise-sustained structures. They also showed that structures sustained by dynamics or by noise have different statistical properties. This is, in particular, reflected by the behavior of their power spectrum which is essentially noise-free in the absolutely unstable regime and presents broadening in the convectively unstable regime. This broadening results from the phase wandering induced by noise amplification. The onset of spectral broadening corresponds to the absolute instability boundary, which may be slightly shifted, according to the noise intensity. As shown by Babcock et al. [5] the experimental results fit nicely with the numerical analysis of the corresponding amplitude equation which is of the complex Ginzburg-Landau type.

The problem of interacting noise-sustained counterpropagating waves was first studied by Deissler and Brand [13]. However, the lack of a detailed stability analysis of individual traveling waves did not allow a complete analysis of the problem. In particular, the distinction these authors make for positive and negative cross couplings between left and right traveling waves does not determine the stability of a traveling wave solution. Nevertheless, they presented qualitatively new results consisting in the possibility of obtaining transitions from convective to absolute instability and vice versa for a given set of parameters. Such transitions can easily be interpreted in the framework of the stability analysis of the uniform and traveling waves states.

It is the aim of this paper to study the effect of spatially distributed noise on convectively unstable systems, either for weak and strong cross couplings between counterpropagating waves, in the presence of group velocity. In Sec. II we introduce the CCGLE and study the linear stability of the uniform reference state and homogeneous traveling wave. We find five different regions with different stability properties in our parameter space. In particular we find that for weak cross coupling there is an intermediate regime between the uniform steady state and the standing wave patterns where traveling waves are convectively unstable. In Sec. III we present our stochastic numerical analysis in the five regions previously identified. We show that, in the weak crosscoupling regime, noise sustained standing waves appear for all values of the bifurcation parameter beyond the Hopf bifurcation. Finally, Sec. IV reports a statistical characterization of the transition between deterministic and noisesustained standing waves in terms of the behavior of an average amplitude, correlation time, and correlation length. An appendix contains details of our numerical procedures.

\section{COUPLED COMPLEX GINZBURG-LANDAU EQUATIONS. STABILITY ANALYSIS OF HOMOGENEOUS STATES}

We consider the CCGLE which describe the dynamics of the amplitudes of two counterpropagating traveling waves with spatial variations in one direction:

$$
\begin{aligned}
\partial_{t} A & (x, t)-v \partial_{x} A(x, t) \\
& =\mu A(x, t)+(1+i \alpha) \partial_{x}^{2} A(x, t) \\
& -(1+i \beta)|A(x, t)|^{2} A(x, t)-(\gamma+i \delta)|B(x, t)|^{2} A(x, t) \\
& +\sqrt{\varepsilon} \xi_{A}(x, t) ; \\
\partial_{t} B(x, t)+v \partial_{x} B(x, t) & \\
& =\mu B(x, t)+(1+i \alpha) \partial_{x}^{2} B(x, t) \\
& -(1+i \beta)|B(x, t)|^{2} B(x, t)-(\gamma+i \delta)|A(x, t)|^{2} B(x, t) \\
& +\sqrt{\varepsilon} \xi_{B}(x, t),
\end{aligned}
$$

where $A(x, t)$ and $B(x, t)$ are the complex amplitudes of the right and left traveling waves. The control parameter $\mu$ measures the distance to the onset of the instability, $v$ is the group velocity. The coefficients $\alpha, \beta, \gamma$, and $\delta$ can be determined from the basic equations of the underlying physical system. A Gaussian, delta correlated, complex white noise of strength level denoted by $\varepsilon, \xi_{j}(x, t), j=A, B$ is assumed to be present in the system. This noise can be spatially distributed or localized (for example, at the inlet of a Taylor-Couette system with through flow). We will consider in this paper a spatially distributed noise with vanishing correlation length (white noise in space and time).

Next we analyze, from a deterministic point of view $(\varepsilon=0)$ the linear stability of homogeneous solutions of Eqs. (2.1).

\section{A. Stability of the uniform reference state}

Linearizing the equations (2.1) around the trivial solution $A(x, t)=B(x, t)=0$, the complex dispersion relation $\omega$ for a disturbance of wave number $K$, that thus behaves as $e^{\omega t+K x}$, becomes:

$$
\omega=\mu+K v+(1+i \alpha) K^{2}, \quad K=k+i q,
$$

and the growth rate of such a perturbation is given by $\operatorname{Re} \omega(K)$. Using the method of steepest descent, the longtime behavior of the system along a ray defined by fixed $x / t$, i.e. in a frame moving with a velocity $v_{0}=x / t$, is governed by the saddle point defined by:

$$
\operatorname{Re}\left(\frac{d \omega}{d K}\right)=v_{0}, \quad \operatorname{Im}\left(\frac{d \omega}{d K}\right)
$$

Since absolute instability occurs when perturbations grow at fixed locations, one has to consider the growth rate of modes evolving with zero group velocity, which are defined by:

$$
\operatorname{Re}\left(\frac{d \omega}{d K}\right)=\operatorname{Im}\left(\frac{d \omega}{d K}\right)=0
$$

These conditions define the following wave number: 


$$
\begin{gathered}
q=-\alpha k, \\
k=-\frac{v}{2\left(1+\alpha^{2}\right)} .
\end{gathered}
$$

The real part of $\omega$, which determines the growth rate $\lambda$ of these modes is then:

$$
\lambda=\operatorname{Re}(\omega)=\mu-\frac{v^{2}}{4\left(1+\alpha^{2}\right)} .
$$

Therefore, the uniform reference state is absolutely unstable if $\lambda>0$. As already shown in [15], this condition determines a critical line in the parameter space which can be expressed for the group velocity $v$ or the control parameter $\mu$ as

$$
v_{c}=2 \sqrt{\mu\left(1+\alpha^{2}\right)} \quad \text { or } \quad \mu_{c}=\frac{v^{2}}{4\left(1+\alpha^{2}\right)} .
$$

Hence, for $0<\mu<\mu_{c}$, the uniform reference state is convectively unstable, and wave patterns are convected away in the absence of noise. For $\mu>\mu_{c}$, wave patterns may grow and are sustained by the dynamics, even in the absence of noise [15].

\section{B. Stability of the uniform traveling wave}

The CGLE (2.1) admits two families of solutions corresponding to traveling waves, $A=\sqrt{\mu-k^{2}} \exp i[k x-(\beta \mu$ $\left.\left.+\alpha k^{2}\right) t\right], B(x, t)=0$, and $B=\sqrt{\mu-k^{2}} \exp i\left[k x-\left(\beta \mu+\alpha k^{2}\right) t\right]$, $A(x, t)=0$. For the sake of simplicity, we first consider uniform solutions $(k=0)$. Without loss of generality, one may study the first family, and, in order to analyze its linear stability, one has to look for solutions in the form $A=(\sqrt{\mu}+a) \exp -i \beta \mu t, B(x, t)=b$, and compute the eigenvalues of the linearized evolution equations for $a, b$, and their complex conjugate. The real parts of the eigenvalues of the Fourier transform of $a$ are well known (see, for example, [7] and [18]) and read:

$$
\begin{gathered}
\operatorname{Re} \omega_{|a|}=-2 \mu-(1-\alpha \beta) q^{2}+\cdots \\
\operatorname{Re} \omega_{\phi}=-(1+\alpha \beta) q^{2}-\frac{\alpha^{2}\left(1+\beta^{2}\right)}{2 \mu} q^{4}+\cdots .
\end{gathered}
$$

The first one, associated with amplitude, is always negative, but the second one, associated with phase, may become positive and the system may experience a Benjamin-Feir instability when $1+\alpha \beta$ is negative $[19,20]$. In the following, we will consider systems where $\alpha$ and $\beta$ are small and positive, such that $1+\alpha \beta>0$.

The only remaining instability mechanism may then result from the growth of $B$. Effectively, the linearized evolution equations for $b$ give the following growth rate:

$$
\omega_{B}=\mu(1-\gamma)-K v+(1+i \alpha) K^{2} .
$$

Hence, in the absence of group velocity, single traveling waves are always stable for $\gamma>1$, while they are unstable for $\gamma<1$ leading to standing wave solutions with $|A|=|B| \neq 0$. The condition $\gamma=1$ thus separates the strong cross-coupling regime $(\gamma>1)$ from the weak cross-coupling regime $(\gamma<1)$. Note that the point $\gamma=0$ does not play any particular role in the stability of traveling waves.

In the presence of a nonvanishing group velocity, traveling waves remain stable for $\gamma>1$, while for $\gamma<1$, they are convectively unstable for $\mu<\mu_{c}^{\prime}$, where $\mu_{c}^{\prime}$ is determined similarly to the preceeding case. Effectively, the conditions

$$
\operatorname{Re}\left(\frac{d \omega_{B}}{d K}\right)=\operatorname{Im}\left(\frac{d \omega_{B}}{d K}\right)=0
$$

define the same wave number

$$
\begin{gathered}
q=-\alpha k, \\
k=-\frac{v}{2\left(1+\alpha^{2}\right)} .
\end{gathered}
$$

However, the real part of $\omega_{B}$, which determines the growth rate of the corresponding modes, is now:

$$
\lambda_{B}=\operatorname{Re}\left(\omega_{B}\right)=\mu(1-\gamma)-\frac{v^{2}}{4\left(1+\alpha^{2}\right)}
$$

and

$$
\mu_{c}^{\prime}=\frac{v^{2}}{4(1-\gamma)\left(1+\alpha^{2}\right)}<\mu_{c}
$$

The corresponding critical group velocity is $v_{c}^{\prime}$ $=v_{c} \sqrt{1-\gamma}$ or

$$
\gamma_{c}=1-\frac{v^{2}}{4 \mu\left(1+\alpha^{2}\right)} .
$$

As a result, on increasing the bifurcation parameter in deterministic systems at fixed $v$ and with $\gamma<1$, traveling waves should be expected between the trivial uniform state and standing waves, as shown on the phase diagram displayed in Fig. 1.

The above deterministic linear stability analysis divides, for $v$ fixed, the $\mu-\gamma$ parameter space in five regions of different spatiotemporal behavior which we label as follows (cf. Fig. 1):

$$
\left.\begin{array}{lll}
\text { 1: } & \mu<\mu_{c} & O \\
\text { 2: } & \mu_{c}<\mu & \mathrm{TW}
\end{array}\right\} \gamma>1,
$$

In the strong cross-coupling regime $(\gamma>1)$ we distinguish two regions. In region 1 the uniform reference state $(O)$ is convectively unstable and it becomes absolute unstable in region 2 where a traveling wave (TW) is absolutely stable. In the weak cross-coupling regime $(\gamma<1)$ we find three regions. In region 3 the uniform reference state $(O)$ is convectively 


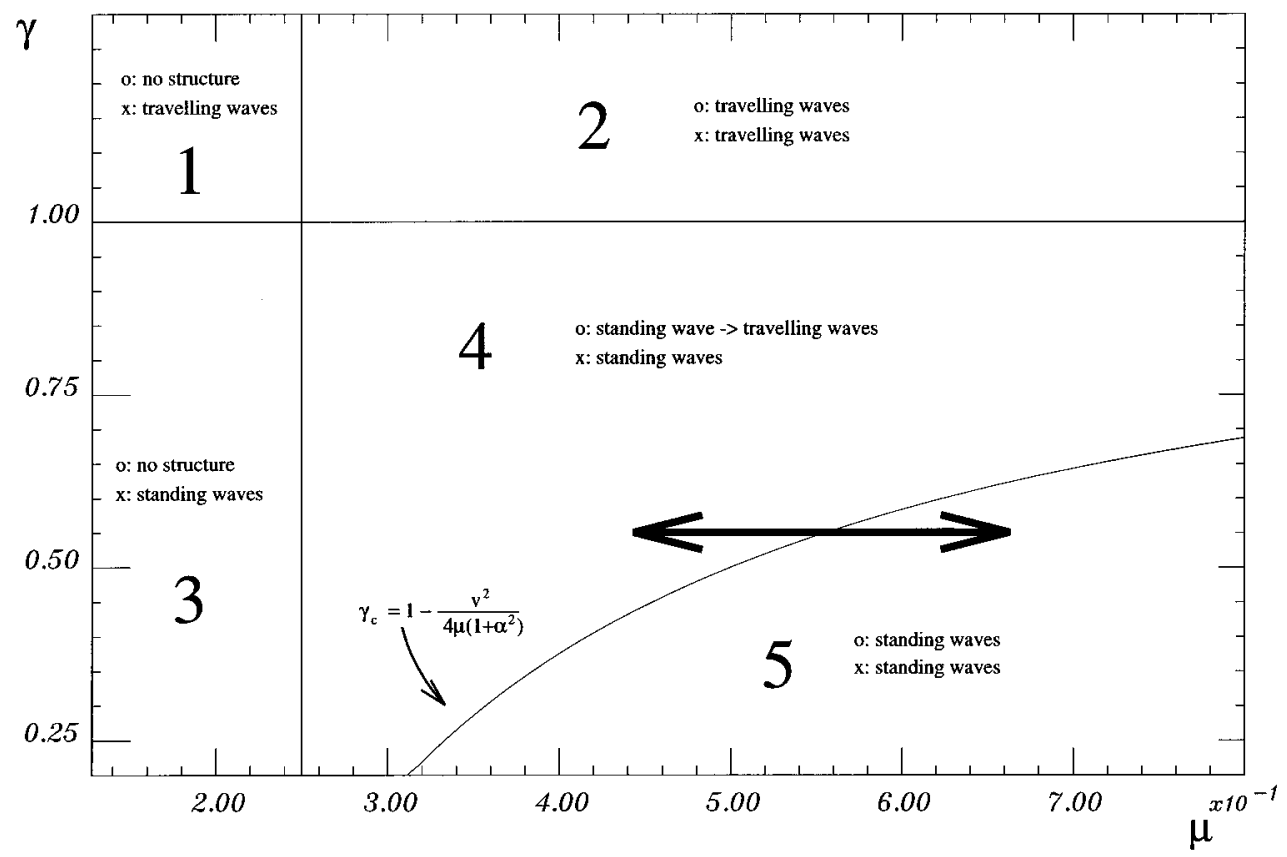

FIG. 1. Stability diagram for homogeneous solutions of Eqs. (2.1). The numbers $1-5$ denote regions of different spatiotemporal behavior, as discussed in the text. o stands for the deterministic case $(\varepsilon=0)$, $\mathrm{x}$ stands for the stochastic case $(\varepsilon \neq 0)$. Parameter values are $\alpha=0.02, \beta=0.04, \delta=0.05$, and $v=1$.

unstable, while in region 4 the traveling wave is the convectively unstable solution. This latter solution becomes absolutely unstable in region 5 where the standing wave (SW) solution is absolutely stable, since we are considering values of $\alpha, \beta$, and $\delta$ sufficiently small to satisfy the standing waves phase stability condition $1+\alpha(\beta-\gamma \delta) /\left(1-\gamma^{2}\right)[10,21]$.

These results can easily be generalized to traveling waves solutions with nonzero wave numbers $(k \neq 0)$. In this case, $a$ perturbations decay if the Benjamin-Feir-Eckhaus criterion $1+\alpha \beta-\left(2 k^{2} / \mu-k^{2}\right)>0$ is satisfied, while $b$ perturbations grow locally when $\mu(1-\gamma)+\gamma k^{2}-v^{2} / 4\left(1+\alpha^{2}\right)$ is positive.

We finally note that the absolute instability criteria derived in this section are a direct consequence of the criterion derived in Sec. II A and Ref. [15], where the linear growth rate of the 0 state, $\mu$, is replaced by its effective linear growth rate $\mu-\gamma|A|^{2}$. This replacement was noted in [13] and it can be interpreted (depending on the sign of $\gamma$ ) as a stabilizing or destabilizing effect of the wave $A$ on the wave $B$. However, as shown above, the sign of $\gamma$ does not determine stability boundaries of the traveling wave solution $A=\sqrt{\mu-k^{2}} \exp i\left[k x-\left(\beta \mu+\alpha k^{2}\right) t\right], B(x, t)=0$.

\section{NOISE-SUSTAINED STRUCTURES}

In this section we analyze numerically the effect of spatially distributed noise on the homogeneous solutions of the CCGLE (2.1). We will explore the parameter space by varying the cross-coupling parameter $\gamma$ and the reduced distance to threshold $\mu$ while keeping the group velocity $v$ set to 1 . We note that this is equivalent to the variation of $\gamma$ and $v$ with $\mu$ fixed, thanks to the scaling

$$
\begin{gathered}
A=\mu^{1 / 2} A^{\prime}, \quad B=\mu^{1 / 2} B^{\prime}, \quad T=\mu^{-1} t, \\
X=\mu^{-1 / 2} x \rightarrow v^{\prime}=\frac{v}{\sqrt{\mu}} .
\end{gathered}
$$

In addition we will fix the noise level to $\varepsilon=0.0001$ and we assign fixed values to the other parameters of (2.1): $\alpha=0.02$, $\beta=0.04$, and $\delta=0.05$. These values belong to the domain of parameters in which the CGLE does not show phase instabilities of the homogenous solutions leading to chaotic behavior.

Noise is expected to be amplified by the convective terms leading to noise-sustained structures in regions where the reference state is deterministically convectively unstable. Therefore, we anticipate that noise effects will result in TW states in the strong coupling regime (regions 1 and 2 of Fig. 1), being noise sustained in region 1. Likewise we anticipate finding SW states in regions 3 , 4, and 5 of Fig. 1 corresponding to weak cross coupling. This implies that noise transforms a $O$ state into a noise-sustained SW in region 3 and a TW state into a noise-sustained SW in region 4 .

In order to check these predictions the stochastic CCGLE (2.1) have been solved numerically with a Heun method (cf. Appendix), random initial conditions around the $A=B=0$ solution, and the following boundary conditions: at the upstream end of each amplitude we use a rigid boundary condition

$$
A(L, t)=0, \quad B(0, t)=0 .
$$

It turned out to be unimportant for the downstream part of the system whether the inlet is fixed or is fluctuating with the noise level [5]. We also checked boundary conditions with a subcritical part $(\mu<0)$ in front of the inlet $(x<0, x>L)$. This influenced only a very small portion of the system near $x=0$ and $x=L$. Different boundary conditions on the outstream end have also a very small influence on the bulk of the system $[5,22]$. We furthermore used a vanishing derivative at the outstream end of each amplitude: 

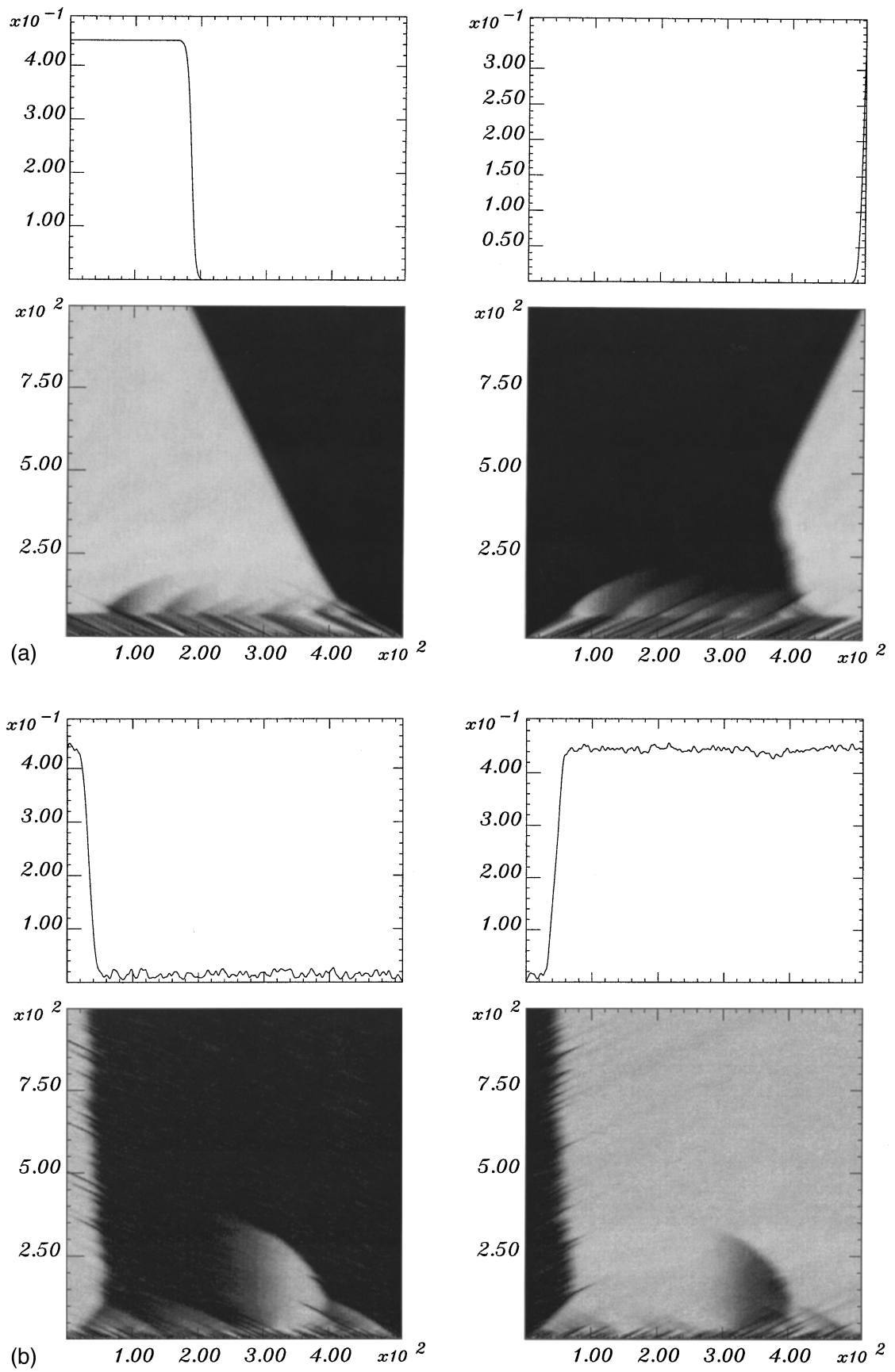

$$
\partial_{x} A(0, t)=0, \quad \partial_{x} B(L, t)=0 .
$$

These boundary conditions mimic extended systems with no reflection of the individual traveling waves at the ends of the system. As such, they rule out the possibility of standing waves maintained by end effects, as it occurs for example in binary fluid convection, even in the absence of noise [23]. In this case, even in the convectively unstable regime, counterpropagating waves, emitted at the boundaries are not entirely convected out of the system since they are partially reflected at each opposite boundary and may thus build an effective but artificial standing wave pattern in the bulk. With the boundary condition used here this effect is absent and no boundary effect should thus interfere with the stochastic effects that we analyze.
FIG. 2. Space (horizontal axis)-time (vertical axis) plot of the moduli of the amplitudes $A(x, t)$ (left) and $B(x, t)$ (right), in arbitrary units, in the region 1 of Fig. $1 \quad(\gamma=1.2$, $\mu=0.165, v=1)$. The upper diagrams show the spatial dependence of $A$ and $B$ at the end of the space-time plots. In this region the trivial state $[A(x, t)=B(x, t)=0]$ is convectively unstable. (a) Deterministic case $(\varepsilon=0)$. The disturbances of the initial random pattern are convected out of the system. (b) Same as (a) but for the stochastic case $(\varepsilon=0.0001)$. Due to the strong cross-coupling $(\gamma>1)$, only a traveling wave structure can survive. The spatially distributed noise effectively sustains the traveling wave structure.

\section{A. Strong cross-coupling, $\gamma>1$}

Due to the fact that the real part of the cross coupling parameter $(\gamma>1)$ between the two fields is larger than the real part of the self coupling $(1+i \beta)$, traveling waves should be the selected pattern in this domain. Due to the symmetry between $A$ and $B$, a competition between these two traveling waves may be observed.

In region 1 , where the group velocity is larger than the critical one $\left(\mu<\mu_{c}\right.$ or $\left.v_{c}<v\right)$ all structures are convected out of the system in the deterministic case $(\varepsilon=0)$, which leads to a stable trivial state with no structure [cf. Fig. 2(a)]. Noise-sustained traveling waves can, however, be observed [cf. Fig. 2(b)]. In the stochastic case one of the traveling waves reaches its saturation value in the bulk and suppresses the other one. There exists a layer at the inlet with a width depending on the distance to the instability $\mu$ and the noise 

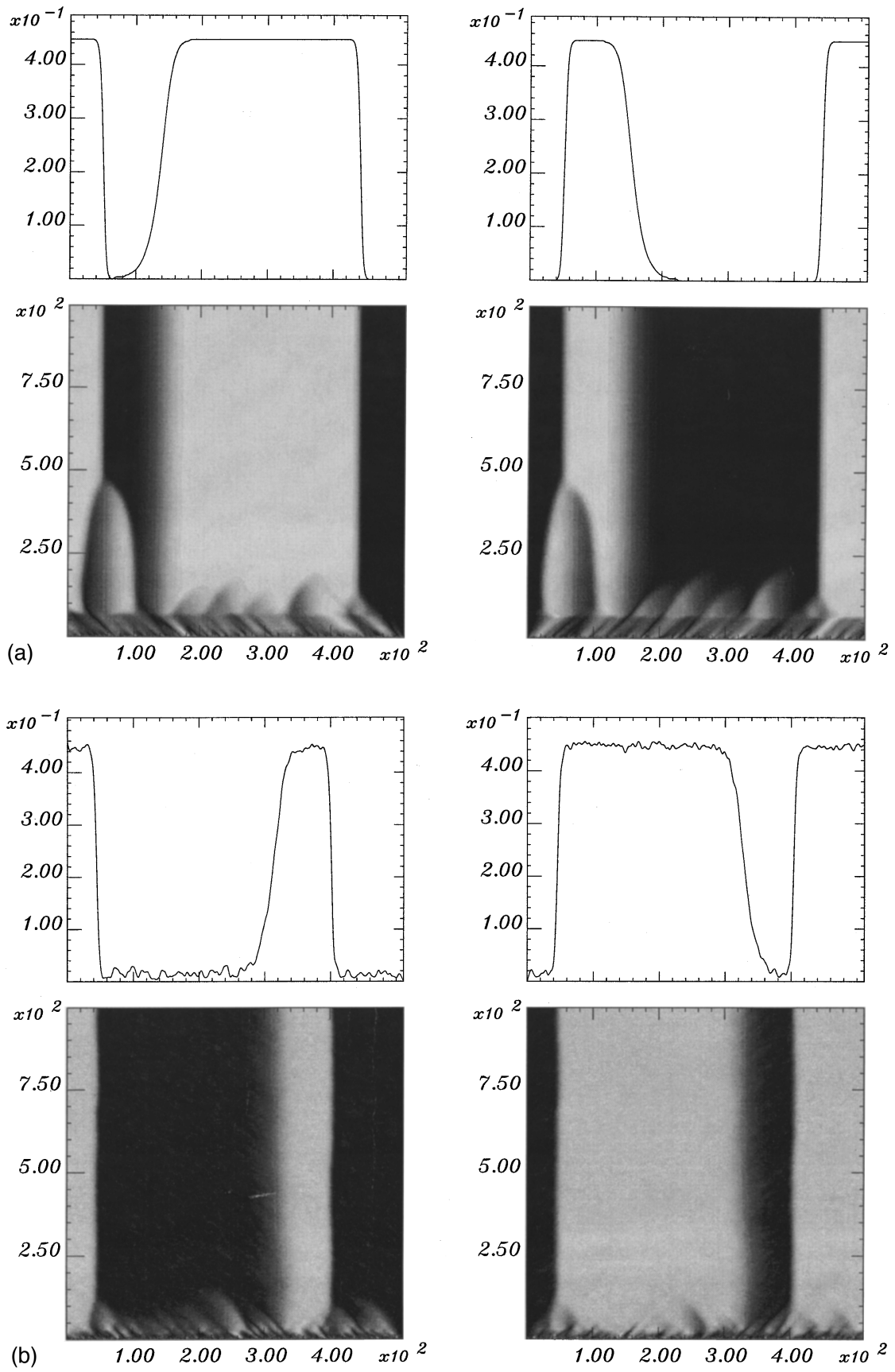

FIG. 3. Same as Fig. 2 in region 2 of Fig. 1 $(\gamma=1.2, \mu=0.8, v=1)$. In this region the trivial state $[A(x, t)=B(x, t)=0]$ is absolutely unstable. The disturbances of the initial random pattern create a traveling wave pattern. The locations of the sources and sinks (fronts between right and left traveling waves) depend on the initial condition as well as on the noise. There is no qualitative difference between the deterministic case (a) $(\varepsilon=0)$ and the stochastic case (b) $(\varepsilon=0.0001)$. level $\varepsilon$. The noise-sustained structure is thus created due to the convectively unstable amplification of the noise as already discussed in $[5,15]$.

In region $2\left(\mu_{c}<\mu\right.$ or $\left.v<v_{c}\right)$ the final states are traveling waves for both cases (deterministic, stochastic). Depending on the initial conditions the system exhibits regions where one of the two traveling waves survives [cf. Figs. 3(a), 3(b)]. Between the right and left traveling wave regions emerge sharp fronts which are called sources and sinks. The motion of these fronts has a very large time scale compared to the emergence of the patterns and have not been studied in this work.

\section{B. Weak cross-coupling, $\gamma<1$}

When the cross-coupling $(\gamma<1)$ is small, the selected patterns should correspond to standing waves. Coexistence of the two traveling waves can be observed. The modulus of the two amplitudes can reach the same value which is the condition for standing waves.

If the group velocity is smaller than the critical value for the absolute instability of the traveling wave state [(region 5) $v<v_{c}^{\prime}$ or $\left.\mu_{c}^{\prime}<\mu\right]$ there is no qualitative difference between the final states, which consist in a standing wave structure [cf. Figs. 4(a), 4(b)].

In the region 4 where the traveling wave state is absolutely stable and the velocity is smaller than the critical velocity for stability of the trivial state $\left(v_{c}^{\prime}<v<v_{c}\right.$ or $\mu_{c}<\mu<\mu_{c}^{\prime}$ ) one observes different spatiotemporal behavior in the deterministic and the stochastic case [cf. Figs. 5(a), $5(\mathrm{~b})]$. In absence of noise $(\varepsilon=0)$, the first transition, during the time evolution, is from a random initial condition to a standing wave structure. Due to front propagation this state 

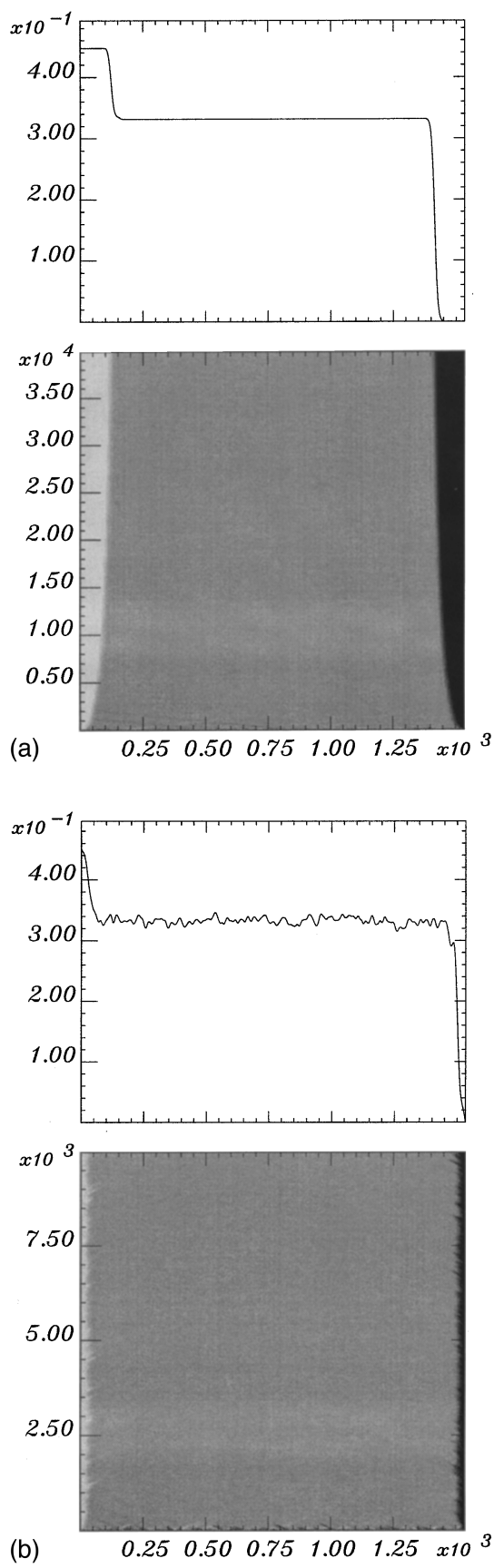
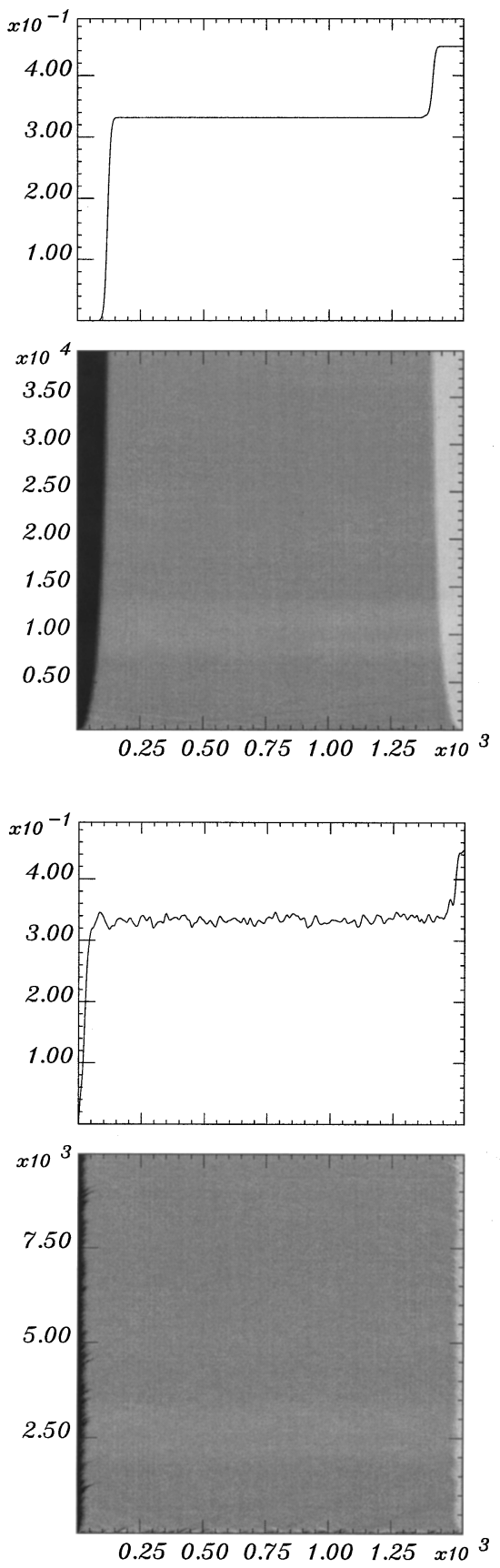

FIG. 4. Same as Fig. 2 in region 5 of Fig. 1 ( $\gamma=0.8, \mu=1.385, v=1$ ). The disturbances of the initial random pattern create a standing wave pattern. There is no qualitative difference between the deterministic (a) $(\varepsilon=0)$ and the stochastic case (b) $(\varepsilon=0.0001)$. is replaced by an exactly symmetric traveling wave structure due to the symmetric boundary conditions [cf. Fig. 5(a)]. If noise effects are included $(\varepsilon \neq 0)$ a standing wave structure is sustained [cf. Fig. 5(b)]. It is important to note that in this case noise changes the nature of the observed spatial structure: In region 1, noise sustains a pattern where there is no pattern with no noise $[5,15]$, but here noise transforms a deterministic TW structure into a noise sustained SW structure.

When the group velocity overcomes the critical value for stability of the trivial state [(region 3) $\mu<\mu_{c}$ or $v_{c}<v$ ] a complicated noise-sustained structure arises. This structure has the main features of a standing wave structure sustained by spatially distributed noise, with a bulk region which fluctuates around the deterministic values for the standing wave solution. From time to time one observes holes (peaks) which are convected through the structure (cf. Fig. 6). Note that the results presented in Fig. 1 of the numerical study performed by Deissler and Brand [13] also correspond to this region of the parameter space where both uniform and traveling wave states are convectively unstable but absolutely stable. Different to us, these authors consider only localized noise at the boundaries. Such a noise source is able to sustain two traveling waves, each of which only fills the half of the system which includes the boundary where it originates. These TWs are convected out of the system when the noise is suppressed. On the contrary, Fig. 2 of [13] presents a situation where, according to our stability analysis, the uniform state is convectively unstable while the traveling wave state is absolutely unstable $\left(\mu_{c}^{\prime}<\mu<\mu_{c}\right.$ with $\mu_{c}^{\prime}<\mu_{c}$ since $\gamma<0)$. In this case, localized noise generates traveling waves which, as the result of their absolute instability, should be 

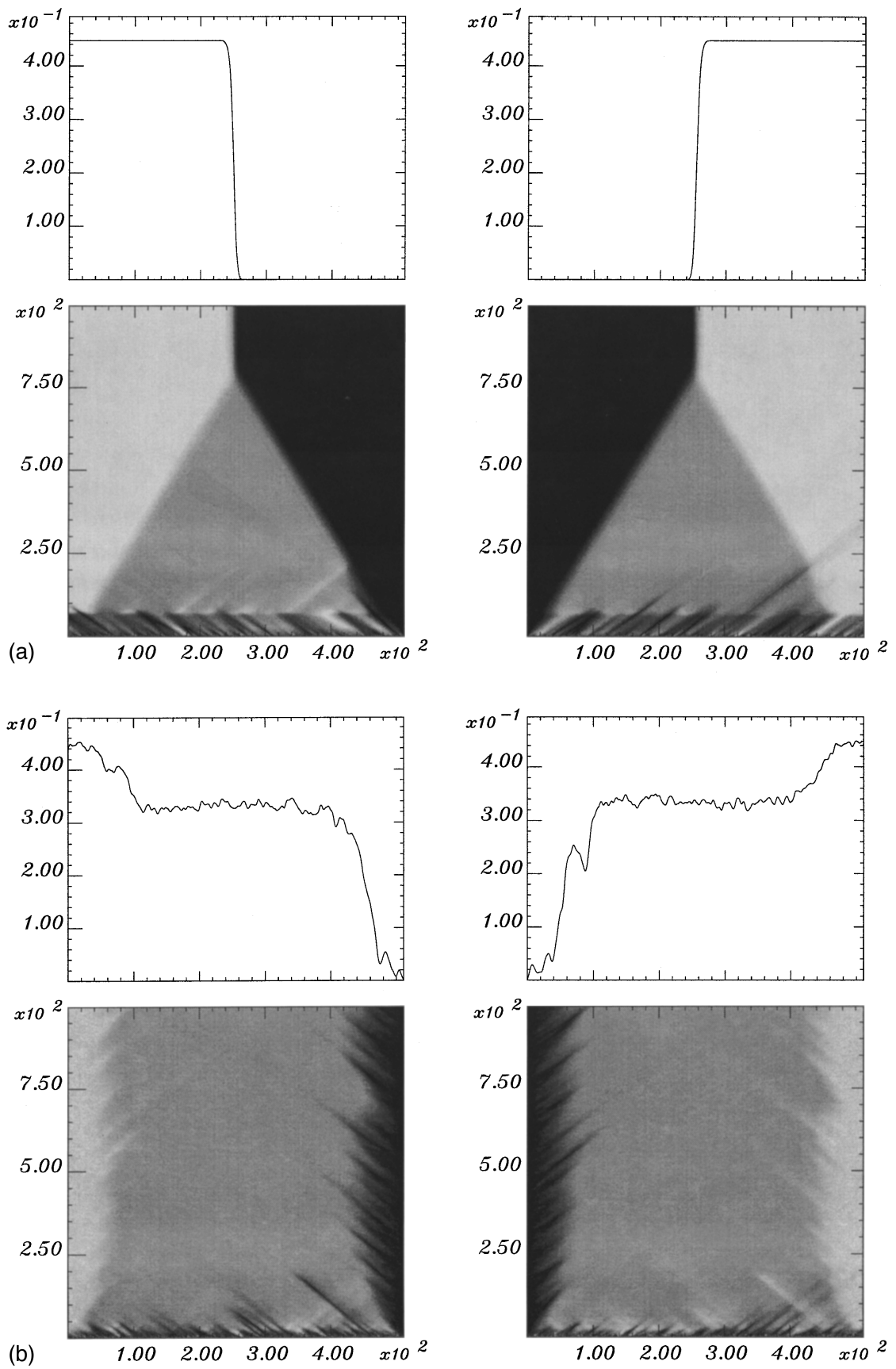
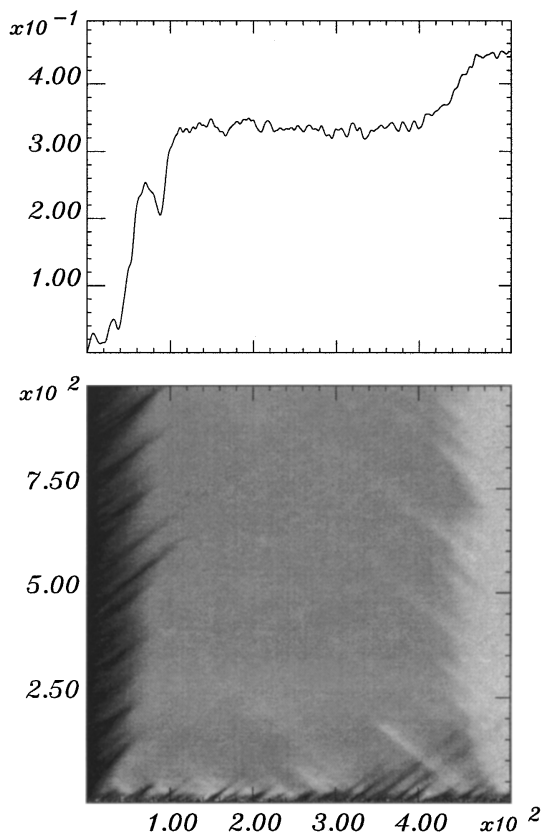

FIG. 5. Same as Fig. 2 in region 4 of Fig. 1 $(\gamma=0.8, \mu=0.408, v=1$ ). (a) Deterministic case $(\varepsilon=0)$ : the disturbances of the initial random pattern create initially a standing wave pattern which is replaced, due to front propagation, by a symmetric traveling wave pattern. (b) Stochastic case $(\varepsilon=0.0001)$ : the spatially distributed noise gives rise, in the bulk of the system, to a noise-sustained standing wave structure fluctuating around the deterministic value. transformed into standing waves which persist after noise removal, in complete agreement with the numerical results of [13].

\section{CHARACTERIZATION OF THE TRANSITION FROM SW TO NOISE-SUSTAINED SW}

The boundary between regions 4 and 5 of Fig. 1 identifies a transition between convective and absolute instability of the traveling wave state (cf. arrow in Fig. 1). When noise is taken into account this transition is transformed into one between a deterministic SW structure and a noisesustained SW. In this section we give a quantitative statistical characterization of this new type of transition investigating the behavior through the transition of different quantities such as amplitudes, correlation time, and correlation length. Our analysis has similarities with the analysis in [5] of the transition between deterministic TW structures and noise-sustained TW (boundary between regions 1 and 2 of Fig. 1).

\section{A. Modulus of the amplitude}

The time average value of the amplitude $A$ at a fixed space point, calculated with no noise $(\varepsilon=0)$, exhibits the deterministic transition between traveling and standing waves at the critical value $\mu_{c}^{\prime}$. In the region of stable standing waves $\left(\mu>\mu_{c}^{\prime}\right)$, the amplitude has its stationary standing wave value $\left\langle|A(x, t)|^{2}\right\rangle=\mu /(1+\gamma)$, which bifurcates to the stationary value of the traveling wave $\left\langle|A(x, t)|^{2}\right\rangle=\mu$ at the critical value $\mu=\mu_{c}^{\prime}$ (cf. Fig. 7). However, the same calculation taking noise into acount shows no trace of the transition: the amplitude has the same averaged value for a deterministic or a noise-sustained standing wave. In order to 

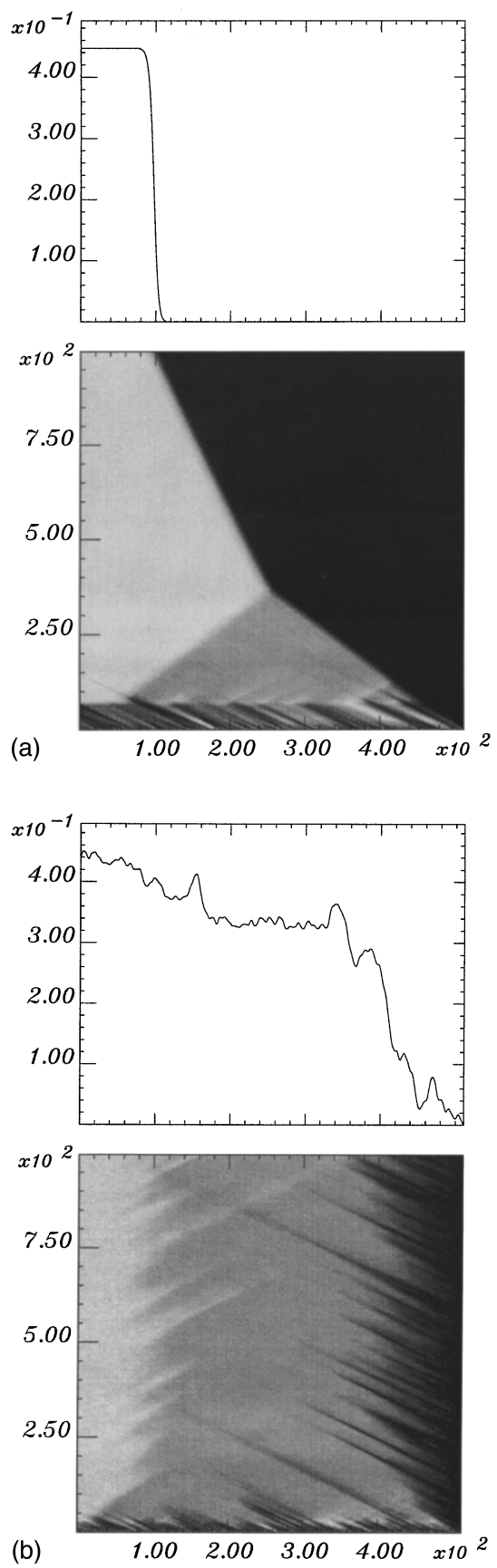
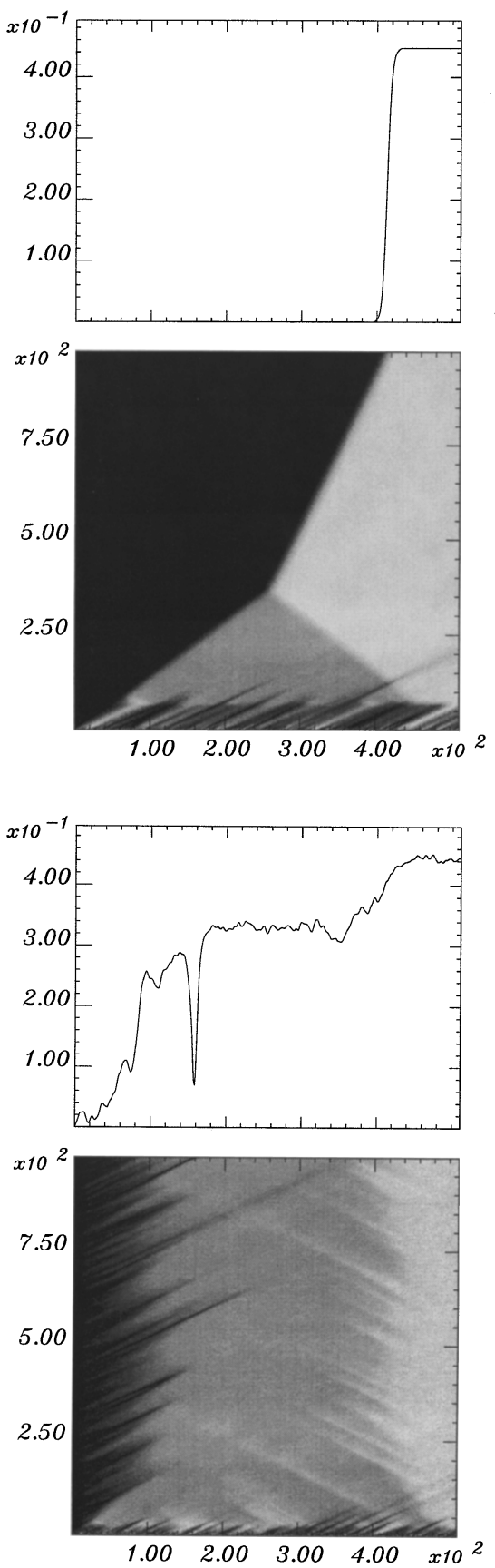

FIG. 6. Same as Fig. 2 in region 3 of Fig. 1 $(\gamma=0.8, \mu=0.165, v=1)$. (a) In the deterministic case the structure is convected out of the system which goes back to the trivial solution. (b) Stochastic case $(\varepsilon=0.0001)$ : a complicated spatiotemporal structure can be observed. The bulk fluctuates around the deterministic value of the standing wave pattern but from time to time a hole (peak) is convected through the pattern. characterize the transition between deterministic and noisesustained standing waves we need to consider quantities giving some information on the degree of temporal or spatial order, as we do next.

\section{B. Correlation time}

We can calculate a correlation time by considering the width of the power spectrum $A\left(x_{0}, w\right)$ of a time series $A\left(x_{0}, t\right)$ at a fixed location $x_{0}$ in the bulk. Since we deal with spatially distributed noise and symmetric boundary conditions, $A\left(x_{0}, w\right)$ is statistically independent of $x_{0}$. We then consider an averaged correlation time $\sigma_{t}^{-1}$ defined in terms of the spatially averaged second moment of the power spectrum:

$$
\bar{\omega}=\left\langle\frac{\int d \omega\left|A\left(x_{0}, \omega\right)\right|^{2} \omega}{\int d \omega\left|A\left(x_{0}, \omega\right)\right|^{2}}\right\rangle,
$$

$$
\sigma_{t}^{2}=\left\langle\frac{\int d \omega\left|A\left(x_{0}, \omega\right)\right|^{2}(\omega-\bar{\omega})^{2}}{\int d \omega\left|A\left(x_{0}, \omega\right)\right|^{2}}\right\rangle,
$$

where $\langle\cdots\rangle$ stands for the spatial average in the interval $0<x<L$.

In Fig. 8 we plot the inverse correlation time $\sigma_{t}$ as a function of $\mu$. The vertical line marks the critical value $\mu_{c}^{\prime}$ 


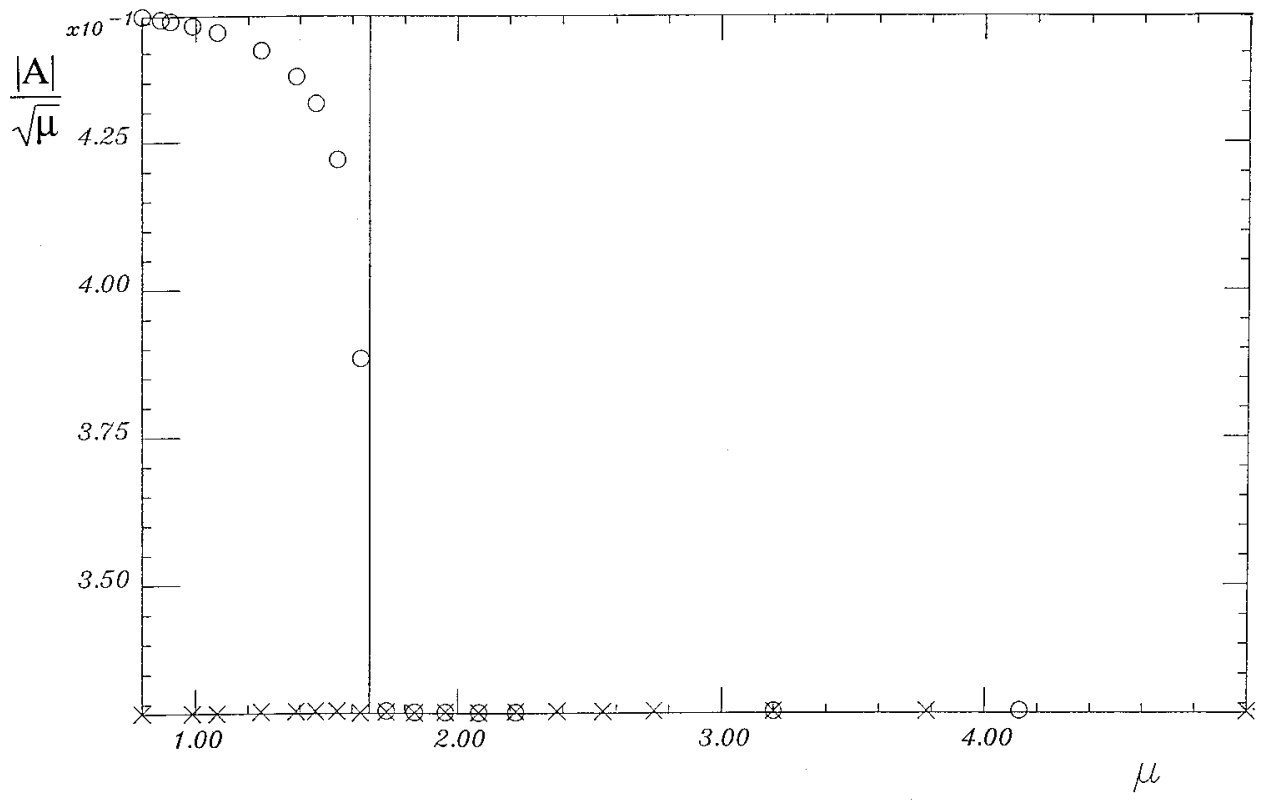

FIG. 7. The time averaged value of $|A| / \sqrt{\mu}$ at a fixed spatial point plotted as a function of the bifurcation parameter for the stochastic $(\mathrm{x})$ and deterministic cases (o). The vertical scale is normalized to the value of $|A|$ for $\mu=0.2$, and the crosscoupling parameter is $\gamma=0.85$. The vertical line denotes the critical control parameter $\mu_{c}^{\prime}$ given by Eq. (2.10). for the instability of the traveling wave solution. We see that no change of behavior is observed in the deterministic case $(\varepsilon=0)$ : a narrow power spectrum exists either for deterministic traveling waves $\mu<\mu_{c}^{\prime}$ or for deterministic standing waves $\mu>\mu_{c}^{\prime}$. However, when noise is taken into account a rather sharp transition is observed between a narrow power spectrum for deterministic standing waves and a wide power spectrum associated with a noise-sustained standing wave. The transition is identified by an apparent divergence of the correlation time $\sigma_{t}^{-1}$. Such divergence occurs for a value of $\mu$ which is shifted with respect to the one identified in a deterministic analysis as the limit for convective instability of traveling waves $\left(\mu=\mu_{c}^{\prime}\right)$. Noise induced shifts of instability boundaries is a rather well known phenomena, and the numerical and experimental results reported in [5] for a transition between regions 1 and 2 of Fig. 1 do not seem to be inconsistent with the possibility of such shift.

\section{Correlation length}

As an alternative characterization we now consider a correlation length $\sigma_{x}^{-1}$ which gives a quantitative characterization of the spatial fluctuations. It is defined in terms of the width $\sigma_{x}$ of the time averaged Fourier spectrum $A(k, t)$ of the amplitude $A(x, t)$ :

$$
\sigma_{x}^{2}=\left\langle\frac{\int d k|A(k, t)|^{2}(k-\bar{k})^{2}}{\int d k|A(k, t)|^{2}}\right\rangle,
$$

where $\langle\cdots\rangle$ stands now for the time average in a large time window $t_{0}<t<t_{0}+T$.

Figure 9 shows the correlation length $\sigma_{x}^{-1}$ under variation of the bifurcation parameter $\mu$. A transition is clearly identi-

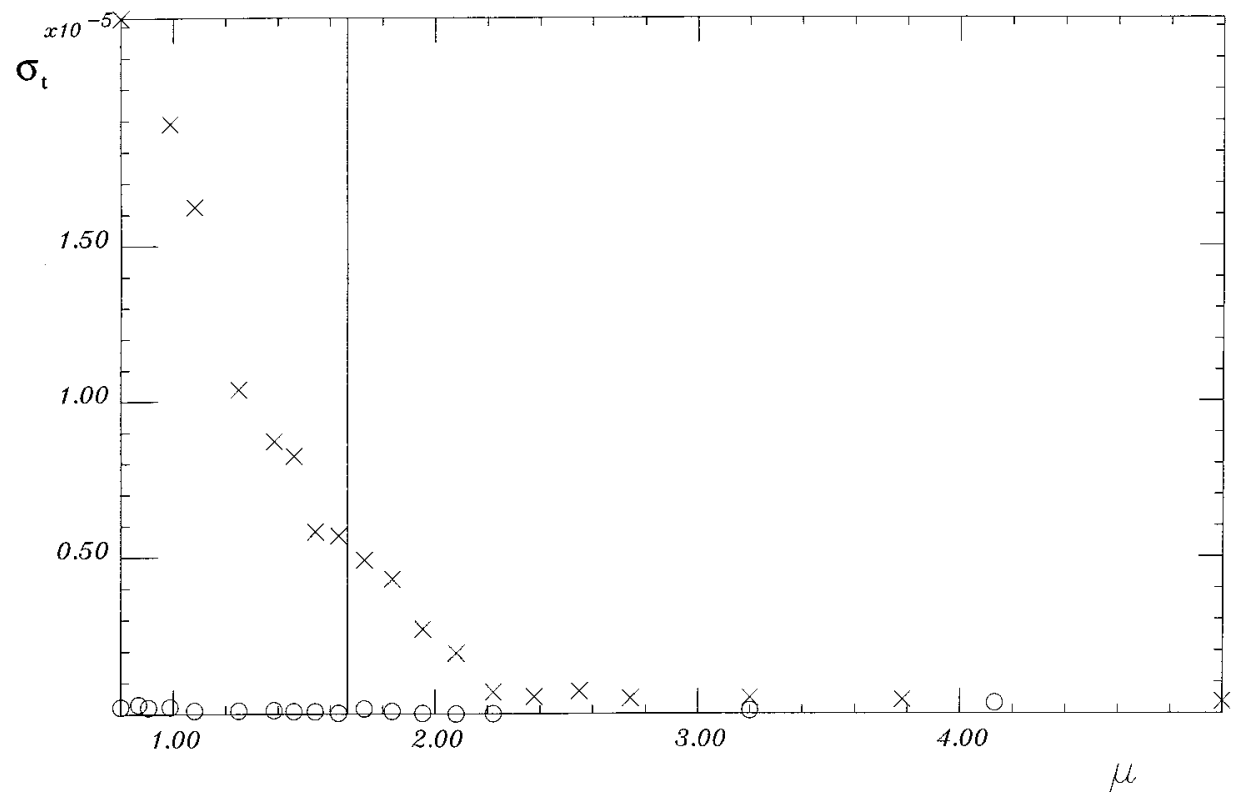

FIG. 8. Width of the power spectrum, in arbitrary units, as given by Eq. (4.1) as a function of the bifurcation parameter for the stochastic (x) and deterministic cases (o). Vertical line as in Fig. 7 and $\gamma=0.85$. 


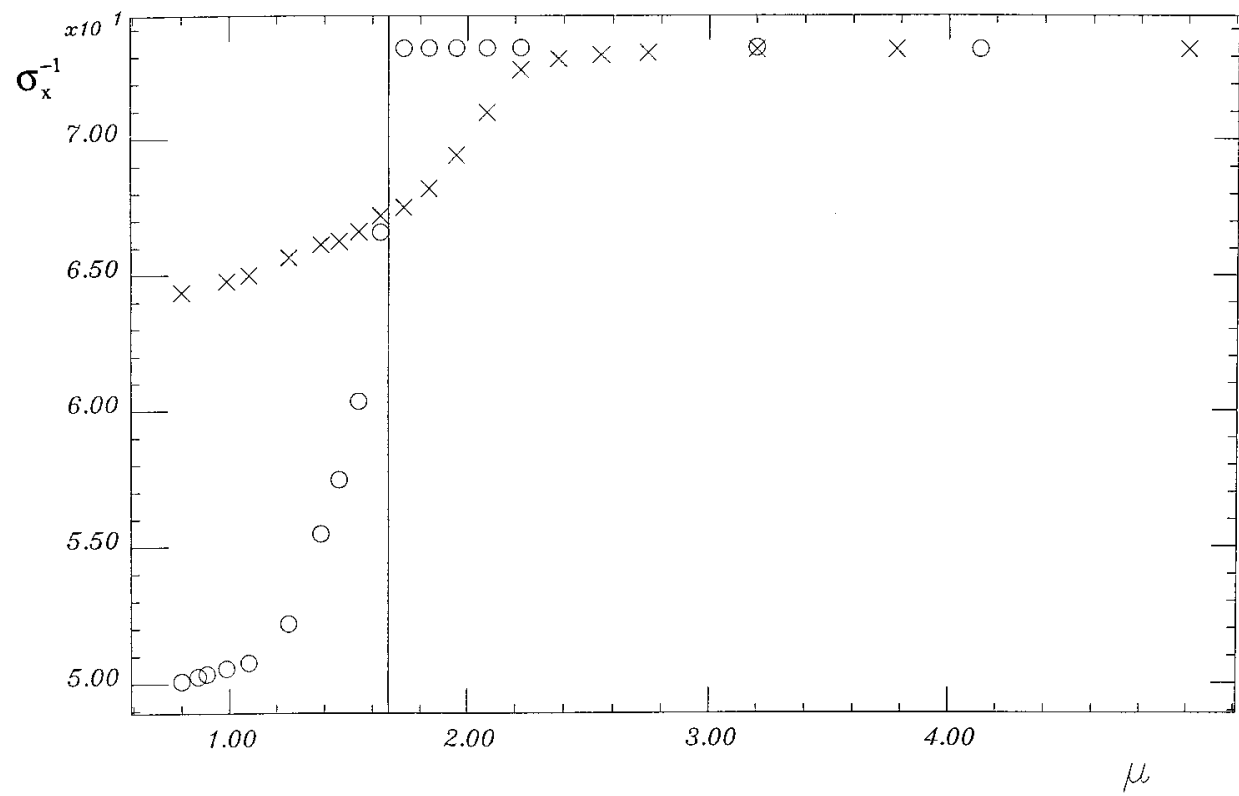

FIG. 9. Correlation length, in arbitrary units, as given by Eq. (4.2) as a function of the bifurcation parameter for the stochastic $(\mathrm{x})$ and deterministic cases (o). Vertical line as in Fig. 7 and $\gamma=0.85$. fied at the same shifted instability point at which the correlation time diverges. At this value of $\mu$ the correlation length saturates here to the system size, while for smaller values of $\mu$ we obtain a smaller correlation length indicating absence of long range order associated with a noise-sustained structure. On the other hand, the deterministic equation $(\varepsilon=0)$ shows a transition exactly at the value $\mu_{c}^{\prime}$ which is predicted in the linear stability analysis (Sec. II). For $\mu>\mu_{c}^{\prime}$ the correlation length also saturates to the system size, while for $\mu<\mu_{c}^{\prime}$ it goes to a value which is a factor $1 / \sqrt{2}$ smaller. This factor is a matter of the definition used for the correlation length of the amplitude $A$. It can be easily understood by noting that for $\mu>\mu_{c}^{\prime}$ the wave with amplitude $A$ fills the whole system in a standing wave state, while for $\mu<\mu_{c}^{\prime}$ it only fills half of the system in the traveling wave state [compare Figs. 4(a) and 5(a)].

\section{CONCLUSION}

In this paper, we have studied the effect of noise on coupled complex Ginzburg-Landau equations on varying not only control parameters such as the distance to threshold $\mu$ or the group velocity $v$, but also the cross coupling $\gamma$ between individual counter-propagating traveling waves. In the strong coupling regime $(\gamma>1)$, we recover the results obtained by other authors, namely, the development of noisesustained traveling waves. In the small coupling regime $(\gamma$ $<1$ ), we show that there is an intermediate region between the trivial uniform state and the standing wave domain where traveling waves are convectively unstable. Our determinisitic numerical analysis confirms this result since one observes, on increasing the bifurcation parameter (or decreasing the group velocity), transitions from the trivial state to traveling waves, and finally to standing waves. In the presence of spatially distributed noise our stochastic numerical analyis shows that sustained standing waves are obtained in all the domain beyond the Hopf bifurcation. Therefore, in the intermediate region mentioned above, noise amplification by the convective terms transforms a traveling wave structure into a noise-sustained standing wave structure. Hence, we conclude that noise is not only able to sustain spatiotemporal patterns, but also to modify pattern selection processes in regimes of convective instability. In addition, we have given a quantitative statistical characterization of the transition between deterministic and noise-sustained standing waves. We have shown that this transition occurs at a noise-shifted point with respect to the one at which traveling waves become absolutely unstable. The transition is identified by an apparent divergence of a correlation time and the saturation of a correlation length to a value given by the system size.

\section{ACKNOWLEDGMENTS}

M.N. was supported by a grant from the Direccion General de Investigacion Cientifica y Tecnica (DGICYT, Spain). D.W. also benefited from the support of the DGICYT, through a grant for a sabbatical stay. We acknowledge financial support from the DGICYT grant PB94-1167. We would also like to thank Raúl Toral for making available a library to create Gaussian distributed random numbers on our computer cluster as well as for his discussions and help during the creation and testing of the numerical integrator. We also acknowledge helpful discussions with $\mathrm{H}$. Brand.

\section{APPENDIX: NUMERICAL METHOD}

The discretized time integration of the CCGLE (2.1) was performed with a second order Runge-Kutta algorithm (Heun method in vector form) [24]

$$
\partial_{t} \mathbf{A}(x, t)=\mathbf{F}(\mathbf{A}(x, t))+\sqrt{\varepsilon} \boldsymbol{\xi}(x, t) .
$$

The Heun recursion relation prescribes 


$$
\begin{gathered}
\mathbf{g}_{1}(x, t)=\mathbf{F}(\mathbf{A}(x, t)), \\
\mathbf{g}_{2}(x, t)=\mathbf{F}\left(\mathbf{A}(x, t)+\Delta t \mathbf{g}_{1}(x, t)+\sqrt{\left.\frac{\varepsilon \Delta t}{(\Delta x)^{n}} \mathbf{u}(x, t)\right),}\right. \\
\mathbf{A}(x, t+\Delta t)=\mathbf{A}(x, t)+\frac{\Delta t}{2}\left[\mathbf{g}_{1}(x, t)+\mathbf{g}_{2}(x, t)\right] \\
+\sqrt{\frac{\varepsilon \Delta t}{(\Delta x)^{n}} \mathbf{u}(x, t),}
\end{gathered}
$$

where $u_{i}(x, t)$ are independent random variables with unit variance and vanishing mean value, and $n$ denotes the dimension of the spatial coordinates. The correlation of the noise variables have the form

$$
\left\langle\xi_{\alpha}^{a}, \xi_{\beta}^{b}\right\rangle=\varepsilon \delta_{\alpha, \beta} \delta_{a, b} \delta\left(x-x^{\prime}, t-t^{\prime}\right),
$$

with $a, b=\operatorname{Re}, \operatorname{Im}$ and $\alpha, \beta=A, B$.
[1] J.J. Niemela, G. Ahlers, and D.S. Cannell, Phys. Rev. Lett. 64, 1365 (1990); K.E. Anderson and R.P. Behringer, Phys. Lett. A145, 323 (1990); P. Kolodner, Phys. Rev. Lett. 66, 1165 (1991).

[2] S. Snyder, Phys. Fluids 11, 728 (1968); C.D. Andereck, S.S. Liu, and H.L. Swinney, J. Fluid. Mech. 164, 155 (1986).

[3] A. Joets and R. Ribotta, in Propagation in Systems far from Thermal Equilibrium, edited by J.E. Wesfreid, H.R. Brand, P. Manneville, G. Albinet, and N. Boccara (Springer, Berlin, 1988).

[4] K.L. Babcock, D.D. Cannell, and G. Ahlers, Phys. Rev. Lett. 67, 3388 (1991); Physica D 61, 40 (1992).

[5] K.L. Babcock, G. Ahlers, and D.S. Cannell, Phys. Rev. E 50, 3670 (1994).

[6] A. Tsameret and V. Steinberg, Europhys. Lett. 14, 331 (1991); Phys. Rev. E 49, 1291 (1994); A. Tsameret, G. Galdner, and V. Steinberg, ibid. 49, 1309 (1994).

[7] M.C. Cross and P.C. Hohenberg, Rev. Mod. Phys. 65, 851 (1993)

[8] H. Haken, Synergetics. An Introduction, 3rd ed. (Springer, Berlin, 1983); Advanced Synergetics, 2nd ed. (Springer, Berlin, 1987).

[9] A.C. Newell, T. Passot, and J. Lega, Annu. Rev. Fluid Mech. 25, 399 (1993).
[10] P. Coullet, S. Fauve, and E. Tirapegui, J. Phys. Lett. 46, L787 (1985).

[11] S. Fauve, in Instabilities and Nonequilibrium Structures, edited by E. Tirapegui (Reidel, Dordrecht, 1987), pp. 69-88.

[12] M. Bestehorn, R. Friedrich, and H. Haken, Z. Phys. 72B, 265 (1988).

[13] R.J. Deissler and H. Brand, Phys. Lett. A 130, 293 (1988).

[14] P. Coullet, T. Frisch, and F. Plaza, Physica D 62, 75 (1993).

[15] R.J. Deissler, J. Stat. Phys. 40, 376 (1985); 54, 1459 (1989); Physica D 25, 233 (1987).

[16] R.J. Deissler, Phys. Rev. E 49, R31 (1994).

[17] J.M. Chomaz, Phys. Rev. Lett. 69, 1931 (1992).

[18] W. van Saarloos and P. C. Hohenberg, Physica D 56, 303 (1992).

[19] T.B. Benjamin and J.E. Feir, J. Fluid Mech. 27, 417 (1967).

[20] A.C. Newell, in Lectures in Applied Mathematics (Am. Math. Society, Providence, 1974), Vol. 15, p. 157.

[21] M. San Miguel, Phys. Rev. Lett. 75, 425 (1995).

[22] M.C. Cross, Phys. Rev. A 38, 3593 (1988).

[23] P. Kolodner, C.M. Surko, and H. Williams, Physica D 37, 319 (1989).

[24] T.C. Gard, Introduction to Stochastic Differential Equations (Marcel Dekker, New York, 1987). 